Subscriber access provided by Lancaster University Library

\title{
Communication
}

\section{A new approach to materials discovery for electronic and thermoelectric properties of single-molecule junctions}

David Zsolt Manrique, Qusiy Hibbeb Al-Galiby, Wenjing Hong, and Colin J. Lambert

Nano Lett., Just Accepted Manuscript • Publication Date (Web): 19 Jan 2016

Downloaded from http://pubs.acs.org on January 19, 2016

\section{Just Accepted}

"Just Accepted" manuscripts have been peer-reviewed and accepted for publication. They are posted online prior to technical editing, formatting for publication and author proofing. The American Chemical Society provides "Just Accepted" as a free service to the research community to expedite the dissemination of scientific material as soon as possible after acceptance. "Just Accepted" manuscripts appear in full in PDF format accompanied by an HTML abstract. "Just Accepted" manuscripts have been fully peer reviewed, but should not be considered the official version of record. They are accessible to all readers and citable by the Digital Object Identifier (DOI®). "Just Accepted" is an optional service offered to authors. Therefore, the "Just Accepted" Web site may not include all articles that will be published in the journal. After a manuscript is technically edited and formatted, it will be removed from the "Just Accepted" Web site and published as an ASAP article. Note that technical editing may introduce minor changes to the manuscript text and/or graphics which could affect content, and all legal disclaimers and ethical guidelines that apply to the journal pertain. ACS cannot be held responsible for errors or consequences arising from the use of information contained in these "Just Accepted" manuscripts. 


\title{
A new approach to materials discovery for
}

\section{electronic and thermoelectric properties of single-}

\author{
David Zsolt Manrique ${ }^{\dagger, *}$, Qusiy Al-Galiby ${ }^{\dagger}$, Wenjing Hong ${ }^{\ddagger, \mathcal{S}}$, Colin J. Lambert ${ }^{\dagger}$ \\ ${ }^{\dagger}$ Department of Physics, Lancaster University, Lancaster LA1 4YB, United Kingdom \\ ${ }^{*}$ Department of Chemical and Biochemical Engineering, College of Chemistry and Chemical \\ Engineering, Xiamen University, Xiamen 361005, China \\ $\S$ Department of Chemistry and Biochemistry, University of Bern, Freiestrasse 3, CH-3012 \\ Bern, Switzerland
}

KEYWORDS. Single molecular junction, quantum circuit, thermopower, conductance, asymmetric and symmetric junctions, thermoelectricity

\begin{abstract}
We have investigated a large set of symmetric and asymmetric molecules to demonstrate a general rule for molecular-scale quantum transport, which provides a new route to materials design and discovery. The rule states "the conductance $\mathrm{G}_{\mathrm{XBY}}$ of an asymmetric molecule is the geometric mean of the conductance of the two symmetric molecules derived from it and the thermopower $\mathrm{S}_{\mathrm{XBY}}$ of the asymmetric molecule is the algebraic mean of their thermopowers". The studied molecules have a structure X-B-Y, where $\mathrm{B}$ is the backbone of the molecule, while $\mathrm{X}$ and $\mathrm{Y}$ are anchor groups, which bind the molecule to metallic electrodes. When applied to experimentally-measured histograms of


conductance and thermopower, the rules apply to the statistically-most-probable values. We investigated molecules with anchors chosen from the following family: cyano, pyridl, dihydrobenzothiol, amine and thiol. For the backbones B, we tested fourteen different structures. We found that the formulae $\left(G_{\mathrm{XBY}}\right)^{2}=G_{\mathrm{XBX}} * G_{\mathrm{YBY}}$ and $S_{\mathrm{XBY}}=\left(S_{\mathrm{XBX}}+S_{\mathrm{YBY}}\right) / 2$ were satisfied in the large majority of the cases, provided the Fermi energy is located within the HOMO-LUMO gap of the molecules. The circuit rules imply that if measurements are performed on molecules with $n_{\mathrm{A}}$ different anchors and $n_{\mathrm{B}}$ different backbones, then properties of $n_{\mathrm{A}}\left(n_{\mathrm{A}}+1\right) n_{\mathrm{B}} / 2$ molecules can be predicted. So for example, in the case of 20 backbones and 10 anchors, 30 measurements (or reliable calculations) can provide a near quantitative estimate for 1070 measurements of other molecules, no extra cost.

Nowadays there exist a variety of techniques for measuring the electrical conductance $G$ and thermopower $S$ of single molecules, such as scanning tunneling microscopy (STM) ${ }^{1,2}$, current probe atomic force microscopy ${ }^{3,}{ }^{4}$, STM-break junction (STM-BJ) ${ }^{5-7}$, crossed-wire geometry $^{8}$, nanoparticle junctions ${ }^{9,}{ }^{10}$, mechanically controlled break junctions(MCBJ) ${ }^{11}$, electromigration setups ${ }^{12,13}$ and nanopores ${ }^{14}$. Schematically, the measured systems are of the form electrode-X-B-Y-electrode, where $\mathrm{X}$ and $\mathrm{Y}$ are anchor groups, which bind the molecule to the electrodes and B is the functional backbone of the molecule. A number of experimental and theoretical studies demonstrated that useful electronic and thermoelectric functionalities, such as switching, sensing, rectifying and heat-to-electricity converters can be optimised by modifying the backbone $\mathrm{B}$ of the molecules, as well as the anchors ${ }^{15-18}$. For molecules of length less than approximately $3 \mathrm{~nm}$, charge transport has been shown to be dominated by phase coherent electron transport ${ }^{19}$, and therefore the subparts $\mathrm{X}, \mathrm{B}$ and $\mathrm{Y}$ cannot be assigned their own conductance or thermopower within the molecule. Nevertheless a recent experimental and theoretical study demonstrated that for molecules containing serially connected meta, para or ortho phenyl rings, their conductances obtained by changing the 
sequencing of the rings are related to each other ${ }^{20}$, which implies that molecular subparts $\mathrm{X}$, $\mathrm{B}$ and $\mathrm{Y}$ are individually characterizable by single numbers. These 'circuit rules' provide a theoretical basis for the systematic categorisation of trends in single-molecule measurement data. They provide guidance for the design and synthesis of molecular devices with optimal electronic and thermoelectric properties by treating molecular components as individual building blocks. In this letter we show that this rule is much more widely applicable than initially suggested in ref. $^{20}$ and can be applied to a very wide range of symmetric and asymmetric molecules, with or without donor and/or acceptor groups. For the first time we also provide a circuit rule for the thermopower $S$ (i.e. Seebeck coefficient) of single molecules. These circuit rules are of interest, because they provide rules for the discovery of new materials by predicting electronic and thermoelectric properties of molecules. This is particularly important, because theoretical methods such as density functional theory and GW many body theory do not usually provide quantitative predictions of such properties ${ }^{21}$.

It is well known that the transmission coefficient of two serially-connected phase-coherent scatterers with individual transmission coefficients $T_{1}$ and $T_{2}$, is of the form $T=$ $\frac{T_{1} T_{2}}{1-2 \sqrt{R_{1} R_{2}} \cos \varphi+R_{1} R_{2}}$, where $\varphi$ is a quantum phase arising from quantum interference (QI) between the scatterers ${ }^{22}$. Consequently the transmission coefficient $T$ cannot normally be factorized to a product of terms associated with the individual scatterers alone. Nevertheless, the following argument leads us to a 'circuit rule' which describes how the transmission changes when the sequential order of the scatterers is changed. First we note that Dyson's equation for the Green's function of a structure comprising three serially-connected subsystems $\mathrm{X}, \mathrm{B}, \mathrm{Y}$ is of the form

$$
\left[\begin{array}{ccc}
E-H_{\mathrm{X}} & -V_{\mathrm{X}} & 0 \\
-V_{\mathrm{X}}^{\dagger} & E-H_{\mathrm{B}} & -V_{\mathrm{Y}} \\
0 & -V_{\mathrm{Y}}^{\dagger} & E-H_{\mathrm{Y}}
\end{array}\right]\left[\begin{array}{ccc}
G_{\mathrm{XX}} & G_{\mathrm{XB}} & G_{\mathrm{XY}} \\
G_{\mathrm{BX}} & G_{\mathrm{BB}} & G_{\mathrm{BY}} \\
G_{\mathrm{YX}} & G_{\mathrm{YB}} & G_{\mathrm{YY}}
\end{array}\right]=I,
$$


where $H_{\mathrm{X}}\left(H_{\mathrm{Y}}\right)$ is the Hamiltonian of the combined left electrode and anchor $\mathrm{X}$ (right electrode and anchor $\mathrm{Y})$ and $V_{\mathrm{X}}\left(V_{\mathrm{Y}}\right)$ is the coupling between the backbone of the molecule and the anchor $\mathrm{X}(\mathrm{Y})$. From this expression, the relevant sub-matrix of the Green's function that describes electron propagation across the molecule from $\mathrm{X}$ to $\mathrm{Y}$ is

$$
G_{\mathrm{YX}}=g_{\mathrm{Y}} V_{\mathrm{Y}}^{\dagger} G_{\mathrm{BB}} V_{\mathrm{X}}^{\dagger} g_{\mathrm{X}}
$$

where $G_{\mathrm{BB}}=\left(E-H_{\mathrm{B}}-\Sigma\right)^{-1}$ is the Green's function of the coupled backbone and $g_{\mathrm{X}}\left(g_{\mathrm{Y}}\right)$ is the Green's function of the combined left electrode and anchor X (right electrode and anchor Y) and $\Sigma=V_{\mathrm{X}} g_{\mathrm{X}} V_{\mathrm{X}}^{\dagger}+V_{\mathrm{Y}}^{\dagger} g_{\mathrm{Y}} V_{\mathrm{Y}}$. If the electrodes are coupled to the anchors through a single site at both ends, the transmission coefficient through the molecule from one electrode to the other is $T_{\mathrm{XBY}}=(\hbar v)^{2}\left|\left[G_{\mathrm{YX}}\right]_{i j}\right|^{2}$, where $v$ is the group velocity of the electrodes, $i$ and $j$ denote the anchor sites connected to electrodes ${ }^{21,23}$. For molecules such as those shown in figure 1 , the anchors are linked to only single sites $k, l$ in the backbone, in which case the transmission coefficients takes the form

$$
T_{\mathrm{XBY}}=\hbar v\left|\left[g_{\mathrm{Y}} V_{\mathrm{Y}}^{\dagger}\right]_{i k}\right|^{2}\left|\left[G_{\mathrm{BB}}\right]_{k l}\right|^{2} \hbar v\left|\left[V_{\mathrm{X}}^{\dagger} g_{\mathrm{X}}\right]_{l j}\right|^{2}=A_{\mathrm{Y}} \times B_{\mathrm{B}} \times A_{\mathrm{X}}
$$

where $A_{\mathrm{X}}=\hbar v\left|\left[V_{\mathrm{X}}^{\dagger} g_{\mathrm{X}}\right]_{l j}\right|^{2}, A_{\mathrm{Y}}=\hbar v\left|\left[g_{\mathrm{Y}} V_{\mathrm{Y}}^{\dagger}\right]_{i k}\right|^{2}$ and $B_{\mathrm{B}}=\left|\left[G_{\mathrm{BB}}\right]_{k l}\right|^{2}$. The factor $B_{\mathrm{B}}$ depends on $\mathrm{X}$ and $\mathrm{Y}$ via the self-energies $V_{\mathrm{X}} g_{\mathrm{X}} V_{\mathrm{X}}^{\dagger}$ and $V_{\mathrm{Y}}^{\dagger} g_{\mathrm{Y}} V_{\mathrm{Y}}$. However if the couplings are sufficiently weak and the Fermi energy does not coincide with the poles of $g_{\mathrm{X}}$ and $g_{\mathrm{Y}}$, then $\Sigma$ can be negligible ${ }^{21}$ and from eq. (2) it follows that

$$
T_{\mathrm{XBY}}^{2}=T_{\mathrm{XBX}} T_{\mathrm{YBY}}
$$

The dependency of $B_{\mathrm{B}}$ of $\mathrm{X}$ and $\mathrm{Y}$ is the smallest when the Fermi energy is located far away from the poles of $g_{\mathrm{X}}$ and $g_{\mathrm{Y}}$, which is the case for off-resonant electron transport in the co- 
tunneling regime. Based on the Landauer formula, and since eq. (3) is valid for a range of energies larger than $k T=25 \mathrm{meV}$, the room temperature conductance also satisfies

$$
G_{\mathrm{XBY}}^{2}=G_{\mathrm{XBX}} G_{\mathrm{YBY}}
$$

Since this approximate relation is true for a range of energies within the HOMO-LUMO gap, the rules for the derivatives of the logarithm of the transmission coefficients are expected to satisfy $\frac{d}{d E} \log T_{\mathrm{XBY}}^{2}=\frac{d}{d E} \log T_{\mathrm{XBX}}+\frac{d}{d E} \log T_{\mathrm{YBY}}$, which leads the circuit rule for the lowtemperature thermopower ${ }^{24}$ :

$$
2 S_{\mathrm{XBY}}=S_{\mathrm{XBX}}+S_{\mathrm{YBY}}
$$

Although density functional theory (DFT) is not a quantitative theory, it has been shown to correctly predict trends in transport properties. In order to demonstrate that the above rules are valid for a wide range of molecular junctions, we performed DFT-based electron transport calculations for 193 molecules. The molecules are classified according to their backbone structures, and their attached anchors (as shown in Figure 1a,b,c). In Figure 1, X and Y indicates the location of the anchor groups. The anchor groups $\mathrm{X}$ and $\mathrm{Y}$ are chosen from the family $\mathrm{CN}, \mathrm{Py}, \mathrm{BT}, \mathrm{NH}_{2}, \mathrm{~S}$, several of which have been studied extensively in the literature ${ }^{25-}$ 32. In Figure 1a, shows 180 molecules formed from 12 backbones and five anchor groups. In the case of the $10 \mathrm{X}-\mathrm{R}-\mathrm{Y}$ molecules shown in Figure $1 \mathrm{~b}, \mathrm{X}$ and $\mathrm{Y}$ are chosen to be one of $\mathrm{CN}, \mathrm{PY}, \mathrm{NH}_{2}, \mathrm{~S}$ and in the case of the $3 \mathrm{X}$-But-Y molecules shown in Figure 1c, $\mathrm{X}$ and $\mathrm{Y}$ are chosen to be either $\mathrm{S}$ or $\mathrm{NH}_{2}$. Figure 1a,b illustrates the anchor as well as the aromatic rings, to which the anchor is attached. For transport calculations, the planar conformations of the molecules were considered, and the gold leads were attached perpendicularly to the plane of the molecules as discussed in the Methods section. Examples of transmission curves for a selection of molecules are shown in Figure 2. 
The circuit formulae are first verified by calculating the room temperature conductance and thermopower, using the DFT predicted Fermi energies for all molecules. To demonstrate the circuit rule for conductance, we separately computed the electrical conductances $\left(G_{\mathrm{XBY}}\right.$, $\left.G_{\mathrm{XBX}}, G_{\mathrm{YBY}}\right)$ and Seebeck coefficients $\left(S_{\mathrm{XBY}}, S_{\mathrm{XBX}}, S_{\mathrm{YBY}}\right)$ of the individual molecules and then plotted the square root of the product $\sqrt{G_{\mathrm{XBX}} G_{\mathrm{YBY}}}$ versus $G_{\mathrm{XBY}}$. In Figure 3a, the small scatter about a straight line demonstrates that in the majority of the cases the circuit rule gives an accurate prediction for the conductance. To demonstrate the circuit rule for thermopower, we separately computed the Seebeck coefficients $\left(S_{\mathrm{XBY}}, S_{\mathrm{XBX}}, S_{\mathrm{YBY}}\right)$ and then plotted the average $\left(S_{\mathrm{XBX}}+S_{\mathrm{YBY}}\right) / 2$ versus $S_{\mathrm{XBY}}$, as shown in Figure $3 \mathrm{~b}$. This remarkable result means that from measurements of the conductances $G_{\mathrm{XBX}}, G_{\mathrm{YBY}}$ it is possible to predict the conductance $G_{\mathrm{XBY}}$ and similarly for the Seebeck coefficients.

We now discuss the sources of deviations from the circuit rule predictions. The inset of Figure $3 \mathrm{a}$, shows that in a number of cases a slight systematic deviation can be observed. The origin of these deviations is illustrated by Figure 2, which shows several different transmission coefficient curves of the $\mathrm{X}-\mathrm{D}_{1}-\mathrm{Y}$ type molecules. These show that if the resonances of the two symmetric molecules are close to each other, then the circuit rule is accurate over almost the whole of HOMO-LUMO gap; for example in Figures 2a,b, the dashed purple curves compare well with the black curves. In these cases $V_{\mathrm{X}} g_{\mathrm{X}} V_{\mathrm{X}}^{\dagger} \approx V_{\mathrm{Y}}^{\dagger} g_{\mathrm{Y}} V_{\mathrm{Y}}$, therefore even if $B_{\mathrm{B}}$ depends strongly on the self energy terms, both sides of the eq. (3) follow the same energy dependence. On the other hand, if the location of the resonances of $\mathrm{X}-\mathrm{B}-\mathrm{X}$ and Y-B-Y differ significantly (from Figure $2 \mathrm{c}$ to $2 \mathrm{~h}$ ), the error in circuit rule for the transmission coefficient is large. In particular cases, when transport through one anchor (such as $\mathrm{X}=\mathrm{NH}_{2}$ ) is $\mathrm{HOMO}$ dominated, whereas the other anchor (such as $\mathrm{Y}=\mathrm{Py}$ ) is LUMO 
dominated, but their HOMO-LUMO gaps are similar, then the two self energy terms $V_{\mathrm{X}} g_{\mathrm{X}} V_{\mathrm{X}}^{\dagger}$ and $V_{\mathrm{Y}}^{\dagger} g_{\mathrm{Y}} V_{\mathrm{Y}}$ are expected to be very different. Such examples can be seen in Figures $2 \mathrm{~g}, \mathrm{~h}$.

We also note that the errors in circuit rule predictions for the thermopower are not necessarily correlated with those of the conductance. While the magnitude of transmission coefficients is inaccurate, the slope of the transmission curve of the $\mathrm{X}-\mathrm{D}_{1}-\mathrm{Y}$ in the log plot in Figure $2 \mathrm{~g}, \mathrm{~h}$ (black curve) remains comparable with the slope of the ones obtained with the circuit rule (dashed purple). In general, $\Sigma$ is the smallest and the circuit rule is most accurate when the Fermi energy is furthest from both the HOMO and LUMO resonances. We note that the DFT calculations typically significantly underestimate the HOMO-LUMO gap ${ }^{33-35}$, therefore for realistic electronic structures the circuit rule may applicable even more accurately than shown in Figure 2 and Figure 3. Other possibilities, that may hinder the accuracy of the circuit rules for realistic conductances, are thermal fluctuations of the molecular conformation and the experimental distributions of junction geometries ${ }^{36}$.

To facilitate the utilisation of the above circuit rules for single-molecule-junction materials discovery, we note that they are a consequence of the fact that the transmission coefficients $T_{X B Y}$ can be factorized into a product of the kind $A_{\mathrm{X}} B_{\mathrm{B}} A_{\mathrm{Y}}$, where $A_{\mathrm{X}}$ and $A_{\mathrm{Y}}$ do not depend on $\mathrm{B}$, but $B_{B}$ may depend on $\mathrm{X}$ and $\mathrm{Y}$. Nevertheless when the Fermi energy is located in the valley of the HOMO-LUMO gap it is possible that $\Sigma$ is negligible and therefore $B_{\mathrm{B}}$ depends only on connectivity and is independent of the choice of the anchor groups. Typically if this is the case, the transmission coefficients can be factorized to independent factors of anchors and backbones, and these factors are transferable between different molecules. In other word, the logarithm of the conductance and the thermopower are sums of transferable factors. To verify this factorizability, we now assume that computed logarithmic conductance values and thermopower values can be written 


$$
\begin{aligned}
& \log _{10} \frac{G_{\mathrm{XBY}}}{G_{0}}=a_{\mathrm{X}}+b_{\mathrm{B}}+a_{\mathrm{Y}} \\
& \frac{S_{\mathrm{XBY}}}{k_{B} / e}=a_{\mathrm{X}}^{\prime}+b^{\prime}{ }_{\mathrm{B}}+a_{\mathrm{Y}}^{\prime}
\end{aligned}
$$

where the factors $a_{\mathrm{X}}, b_{\mathrm{B}}, a_{\mathrm{Y}}, a_{\mathrm{X}}^{\prime}, b^{\prime}{ }_{\mathrm{B}}, a_{\mathrm{Y}}^{\prime}$ are independent and transferable. To obtain these parameters, we minimize numerically the function $F=\sum_{\mathrm{B}}^{\mathrm{Backbones}} \sum_{\mathrm{X}}^{\mathrm{Anchors}} \sum_{\mathrm{Y} \geq \mathrm{X}}^{\mathrm{Anchors}}\left(a_{\mathrm{X}}+\right.$ $\left.b_{\mathrm{B}}+a_{\mathrm{Y}}-\log _{10} \frac{G_{\mathrm{XBY}}}{G_{0}}\right)^{2}$, where the $G_{\mathrm{XBY}}$ values are the DFT-computed conductance values (see Methods section). We note that the separation between the anchor and backbone terms is arbitrary, therefore we set the backbone term of X-RR-Y type molecules to zero, i.e. we choose $b_{\mathrm{RR}}=0$, and in this calculation we choose the molecules in Figure 1a only. With this choice the $a_{\mathrm{X}}$ terms parametrize the anchoring structures shown in Figure 1a and Table 1, that is the anchor plus the aromatic ring. Consequently the $b_{\mathrm{B}}$ terms parametrize the backbone, that is the inner part of the molecules between the aromatic rings in Figure 1a. From $n_{\mathrm{A}}=5$ different anchors and $n_{\mathrm{B}}=12$ different backbones, this procedure yields $5 a_{\mathrm{X}}$ parameters and $12 b_{\mathrm{B}}$ parameters, from which we can reproduce the logarithmic conductance of $n_{\mathrm{A}}\left(n_{\mathrm{A}}+1\right) n_{\mathrm{B}} / 2=180$ molecules. Similarly, the same minimizing procedure is used to obtain the thermopower parameters $a_{\mathrm{X}}^{\prime}$ and $b_{\mathrm{B}}^{\prime}$. Table 1 shows the anchor and backbone parameters obtained using the above minimizing procedure. To demonstrate that they can be used to predict coductances and thermopowers, Figure 4a shows a comparison between the sum $a_{\mathrm{X}}+b_{\mathrm{B}}+a_{\mathrm{Y}}$, and the conductance $G_{\mathrm{XBY}}$ while Figure $4 \mathrm{~b}$ compares $a_{\mathrm{X}}^{\prime}+b_{\mathrm{B}^{\prime}}^{\prime}+a_{\mathrm{Y}}^{\prime}$ with $S_{\mathrm{XBY}}$. In Figure $4 \mathrm{a}$, for each molecule, for the DFT computed conductance value $G_{\mathrm{XBY}}$ on the horizontal axis, we plotted the corresponding $10^{a_{\mathrm{X}}+b_{\mathrm{B}}+a_{\mathrm{Y}}} G_{0}$ value marked by a red cross. The fact that the majority of the red crosses are close to the diagonal black line shows that the $5 a_{\mathrm{X}}$ parameters and $12 b_{\mathrm{B}}$ parameters in Table 1 can reproduce the logarithmic conductance of the 180 molecules in figure 1a accurately. In essence, by minimizing the function $F$ with 
respect to the $5 a_{\mathrm{X}}$ parameters and $12 b_{\mathrm{B}}$ parameters, we obtain their optimal value which holds the information of the conductance values of the 180 molecules. This is possible only because the conductance can be factorized with good accuracy according to eq. (3) and therefore the 180 molecular conductances are not independent. Similarly, in Figure 4b, for each molecule, for the DFT computed thermopower value $S_{\mathrm{XBY}}$ on the horizontal axis, we plotted the corresponding $\left(a_{\mathrm{X}}^{\prime}+b^{\prime}{ }_{\mathrm{B}}+a_{\mathrm{Y}}^{\prime}\right) S_{0}$ value marked by a red $\mathrm{x}$. It is interesting to note that for the conductance, the values of anchor parameter $a_{X}$ in Table 1 vary less than the values of the backbone parameters $b_{\mathrm{B}}$. This is in contrast with the thermopower, for which the magnitudes of the anchor parameters $a^{\prime}{ }_{\mathrm{X}}$ vary more than the magnitudes of the backbone parameters $b^{\prime}$, which suggests that the anchor may play a more dominant role in controlling the thermopower than the conductance.

Having demonstrated the validity of the circuit rules, within a consistent set of DFT-based calculations, we now discuss how they can be used experimentally for real-world discovery of single-molecule junction properties. Although DFT is widely used for analysing electron transport in single molecule junctions, it is at best a qualitative theory and therefore for accurate utilisation of the rules, the parameters $a_{\mathrm{X}}, b_{\mathrm{B}}, a_{\mathrm{Y}}$ and $a_{\mathrm{X}}^{\prime}, b^{\prime}{ }_{\mathrm{B}}, a_{\mathrm{Y}}^{\prime}$ should be determined experimentally. In a typical break junction experiment, the measured value of $G_{\mathrm{XBY}}$ varies markedly from measurement to measurement, because of variability in the atomic arrangement of the electrodes and in the electrode-anchor group binding geometry. Consequently many (often thousands) of conductance measurements are made and histograms of the logarithmic conductance $g_{\mathrm{XBY}}=\log _{10} G_{\mathrm{XBY}} / G_{0}$ are constructed. If $\bar{g}_{\mathrm{XBY}}$ is the most probable value of such a histogram, then the experimentally-quoted value is $\bar{G}_{\mathrm{XBY}} / G_{0}=10^{\bar{g}_{\mathrm{XBY}}}$. This variability is reflected in the anchor parameters $a_{\mathrm{X}}, a_{\mathrm{Y}}$. Therefore when applying the circuit rule to such experiments, it should be applied in a statistical sense. 
If the most probable values of $a_{\mathrm{X}}, a_{\mathrm{Y}}$ are $\bar{a}_{\mathrm{X}}, \bar{a}_{\mathrm{Y}}$, then the most probable value of $\log _{10} \frac{G_{\mathrm{XBY}}}{G_{0}}$ is $\bar{g}_{\mathrm{XBY}}=\bar{a}_{\mathrm{X}}+b_{\mathrm{B}}+\bar{a}_{\mathrm{Y}}$. Conversely, when the fitting procedure of equation (5) in 'Methods' is carried out using experimentally-quoted values, the resulting parameters are $\bar{a}_{\mathrm{X}}, b_{\mathrm{B}}, \bar{a}_{\mathrm{Y}}$, rather than $a_{\mathrm{X}}, b_{\mathrm{B}}, a_{\mathrm{Y}}$. This is another reason why it is unsafe to use DFT values to make quantitative predictions, because it is usually much too expensive to simulate conductance histograms and therefore typically conductances of only a few anchoring condifgurations are reported.

At present we are aware of only one set of measurements on both asymmetric (X-B-Y) and symmetric (X-B-X and Y-B-Y) molecules with the same backbone. In ref ${ }^{37}$ the measured conductance values of molecules S-R-S, $\mathrm{NH}_{2}-\mathrm{R}-\mathrm{NH}_{2}$ are found to be $0.012 G_{0}, 0.005 G_{0}$ respectively and for the asymmetric $\mathrm{NH}_{2}-\mathrm{R}-\mathrm{S}$ molecule two conductance values $0.006 G_{0}$ and $0.009 G_{0}$ were reported. The circuit rule gives $G / G_{0}=0.008$, which compares well with both of the measured values, thereby providing a direct experimental verification of the circuit rule. To illustrate how the circuit rules can be used to predict experimental conductances for future molecules from measured values of molecules available in the literature, we make use of the above factorisation procedure. To perform the fitting, we collected measured conductances for 19 different molecules from the literature $20,25,31,38-40$ and used these to characterize 5 anchors and 5 backbones. In Table 2 the anchor and backbone parameters are listed characterized with the experimental conductances. The parameters obtained from the experimental values are smaller than the theoretical values, as expected, due to the DFT's systematic errors with underestimating the HOMO-LUMO gap ${ }^{35,41}$ and the neglect of environmental and thermal effects in the calculations ${ }^{36}$. Figure $4 \mathrm{a}$ and Table 3 show that the characterisation can reproduce the conductance of the 19 different molecules with good accuracy (marked with green triangles). The selection covers the typical experimental range of conductances from $10^{-2} G_{0}$ to $10^{-6} G_{0}$. Usually the short molecules with higher conductance, 
due to the snap back effect in the break-junction methods, are difficult to measure reliably. The longer molecules with lower conductances (below $10^{-6} G_{0}$ ), due to the instrumental sensitivity, are also difficult to measure. The $X-T_{n}-X$ type molecules are from ref ${ }^{40}$, where $X$ $=\mathrm{Py}, \mathrm{NH}_{2}, \mathrm{~S}, \mathrm{BT}$ and $\mathrm{CN}$, and $\mathrm{n}=1,2$ and 4 , but varies for different $\mathrm{X}$ because not all molecules could been synthetized. The conductances for the Py-OPE-Py and Py-OPE(Meta)Py type molecules are from $\operatorname{ref}^{20}$ (Py-OPE(Meta)-Py denotes pmp), for the molecule $\mathrm{NH}_{2}$ OPE- $\mathrm{NH}_{2}$ is from $\mathrm{ref}^{39}$ and S-OPE-S molecule is from ref $^{26}$. From ref ${ }^{29}$ we obtained the conductance of $\mathrm{NH}_{2}-\mathrm{RR}-\mathrm{NH}_{2}$ and from ref ${ }^{31} \mathrm{Py}-\mathrm{RR}-\mathrm{Py}$. The precise conductance values used in the calculations are quoted in Table 3. Finally we note that in Figure 4a, the green triangles, and Table 2, 3 and 4 are based on experimental conductance values, which naturally includes the effect of fluctuations. The fact that the green triangles are close to the diagonal demonstrates that the circuit rule indeed applies to 19 different experimentallyquoted most-probable conductances.

In conclusion, we have demonstrated that for a large variety of molecules of the type X-B-Y with different backbones B and anchors X,Y, the molecular conductance and thermopower of asymmetric molecules can be obtained as geometric and algebraic averages of the zero-bias conductances and thermopowers of their symmetric counterparts respectively. At a fundamental level, a requirement for the validity of this 'circuit rule' is that the parts $\mathrm{X}, \mathrm{B}$ and Y should be weakly coupled, so that multiple scattering effects contained in the selfenergy $\Sigma$ in equation (1) can be neglected. This also requires that the Fermi energy should be located within the HOMO-LUMO gap of the molecules and therefore transport should be 'off-resonance' and take place via coherent tunnelling. The validity of this circuit rule is demonstrated through DFT calculations on 193 molecules, which confirms that the rule applies to molecules exhibiting off-resonance transport. In our experience of comparing theory with measurements of single-molecule electron transport, we have found that in 
almost all cases, unless an electrostatic or electrochemical gate is applied, transport does not take place near resonances and therefore the rule can be expected to have wide applicability. The derivation of the rule assumes that transport takes place via coherent tunneling and that inelastic effects are negligible. Experiment demonstrates that the length scale for the onset of significant inelastic scattering at room temperature can be of order $3 \mathrm{~nm}^{19,42}$. All the molecules in our study are shorter than this length. Furthermore comparison with 19 experimental molecular conductance values from the literature, originating from different laboratories shows that the circuit rules can be applied successfully at room temperature. Finally we note that as demonstrated in refs ${ }^{43,44}$ the backbone contribution $B_{\mathrm{B}}$ (see eq. (2)) depends on the points of contact $k, l$ of the anchors to the backbone and therefore the circuit rule should be applied only to families of molecules containing backbones with the same connectivity to the anchors or equivalently, backbones with different connectivities should be treated as distinct entities, which means for example that a meta-connected backbone is distinct from a para-connected backbone as values of $b_{\mathrm{B}}$ of OPE and OPE(meta) show in Table 2.

For a workable thermoelectric device one needs to increase both the Seebeck coefficient $S$ and the electrical conductance $G$ and simultaneously minimise the thermal conductance. The proposed circuit rule addresses the first two challenges by predicting $S$ and $G$ of hitherto unsynthesized molecules, thereby avoiding unnecessary synthetic effort. The rule also provides new insight and chemical intuition by organising a large body of information. The utility of the rule for 'materials discovery' derives from the potential translation of singlemolecule functionality into thin molecular films, formed from a monolayer of molecules is sandwiched between a planar bottom electrode and a planar top electrode, in which the current/heat flows through the moelcules from the bottom to the top electrode. The question of how single-molecule properties translate into thin films is non-trivial and beyond the scope 
of this paper. However knowledge of transport properties at the single molcule level will surely inform our understanding of such thin-film materials.

For other properties, the rules can be used to obtain inequalities. For example, they imply that if $S_{\mathrm{XX}}$ and $S_{\mathrm{YY}}$ have the same sign then the powerfactor $P_{\mathrm{XY}}=G_{\mathrm{XY}} S_{\mathrm{XY}}^{2}$ is bounded by the power factors of the symmetric molecules, that is $P_{\mathrm{YY}} \leq P_{\mathrm{XY}} \leq P_{\mathrm{XX}}$ and if $S_{\mathrm{XX}}$ and $S_{\mathrm{YY}}$ have opposite sign, the powerfactor will be diminished significantly. To aid utilisation of the rules for the discovery of new junction properties, we also demonstrated that conductances and thermopowers can be characterized by transferable anchor and backbone parameters. Such characterization can be used to identify optimally functioning molecular devices for future synthesis. The accuracy of this characterization was demonstrated using experimental conductances of 19 different molecules from the literature.

Methods. In order to demonstrate computationally the circuit formulas, we performed DFT based electron transport calculations on systematically constructed gold-molecule-gold systems. All together 193 molecules were constructed with attached gold pyramids as electrodes, with anchors and backbones showed in Figures 1a,b,c. To consistently attach the pyramids to the molecules with many different backbones and anchors, we first prepared a relaxed molecular geometry with their planar and extended conformations. The relaxation was performed with MOPAC2012 RHF method and PM7 parameter-set ${ }^{45}$ with constraints that kept the molecular conformations planar. During the relaxation, the non-hydrogen atoms were allowed to move only in the molecular plane and only the hydrogen atoms were allowed to move away from the molecular plane. The initial conformation for the relaxation was linear extended where the anchors are at the two far ends of the molecule modelling a possible conformation within an break-junction experiment (shown in Figure 5a) when a typical conductance plateau is recorded ${ }^{27,}{ }^{46}$. This is typically occurring when the gap between the electrodes is slightly less than the molecular length, illustrated in Figure 5a. In 
the case of thiol, the sulphur was capped with a hydrogen atom for the relaxation, then after the relaxation the hydrogen atom from the sulphur was removed. We performed relaxations with SIESTA ${ }^{47}$ DFT code (force tolerance $=0.01$, GGA, DZP) as well for a few molecules and found that planar conformations are usually stable, with significant exceptions of X-RRY type molecules, where the aromatic rings are twisted away from each other. Since our investigation focuses on the electronic structures, we consistently kept the all structures planar. For the planar molecular structures a 35-atom 111 directed gold pyramid was attached to the anchoring atoms ( $\mathrm{S}$ and $\mathrm{N}$ ) perpendicularly to the molecular plane. The apex gold atom-anchor atom distance was set $2.1 \AA$ for the Py, $\mathrm{CN}$ anchors, $2.3 \AA$ for the $\mathrm{NH}_{2}$ and BT and $2.35 \AA$ for the thiol anchor. This setup allowed consistent systematic comparisons between molecules with different backbones and anchors. The electronic transport calculations were performed by first obtaining the Hamiltonian of the isolated Au-molecule$\mathrm{Au}$ structure by SIESTA, DZP basis set and GGA-PBE exchange-correlation potential parameterization. Then the obtained Hamiltonian was used in GOLLUM ${ }^{48}$, with wide-band approximation to calculate the transmission coefficients, the room temperature conductance and the room temperature thermopower with the DFT computed Fermi energy. The wideband lead was attached to the two outer layers of the gold pyramid with $\Gamma=4.0 \mathrm{eV}$ coupling ${ }^{49}$. The anchor and backbone parameters were determined by minimizing numerically the function

$$
F=\sum_{\mathrm{B}}^{\text {Backbones Anchors Anchors }} \sum_{\mathrm{X}} \sum_{\mathrm{Y} \geq \mathrm{X}}\left(a_{\mathrm{X}}+b_{\mathrm{B}}+a_{\mathrm{Y}}-\log _{10} \frac{G_{\mathrm{XBY}}}{G_{0}}\right)^{2}
$$

with respect to the various $a_{\mathrm{X}}, b_{\mathrm{B}}$ parameters with the constraint $b_{\mathrm{RR}}=0$, using BroydenFletcher-Goldfarb-Shanno (BFGS) method, where 'Backbones' and 'Anchors' are in figure 
1a, $G_{\mathrm{XBY}}$ is the DFT-computed conductance value and $\mathrm{Y} \geq \mathrm{X}$ denotes the exclusion of the double counting the same molecule.

\section{FIGURES}

a)
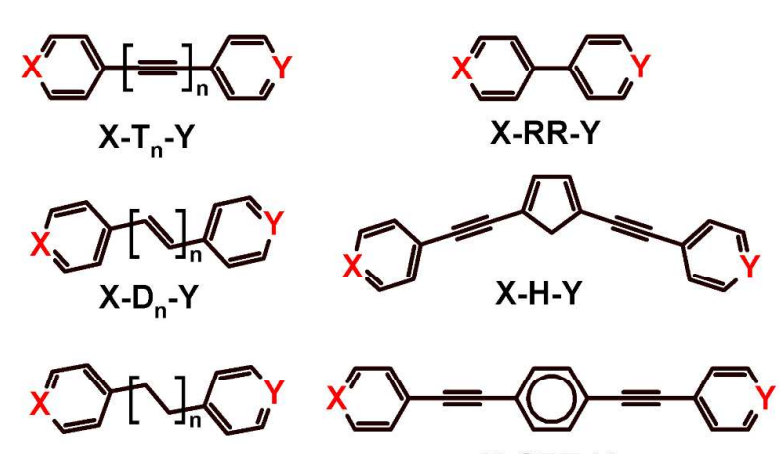

$X-S_{n}-Y$
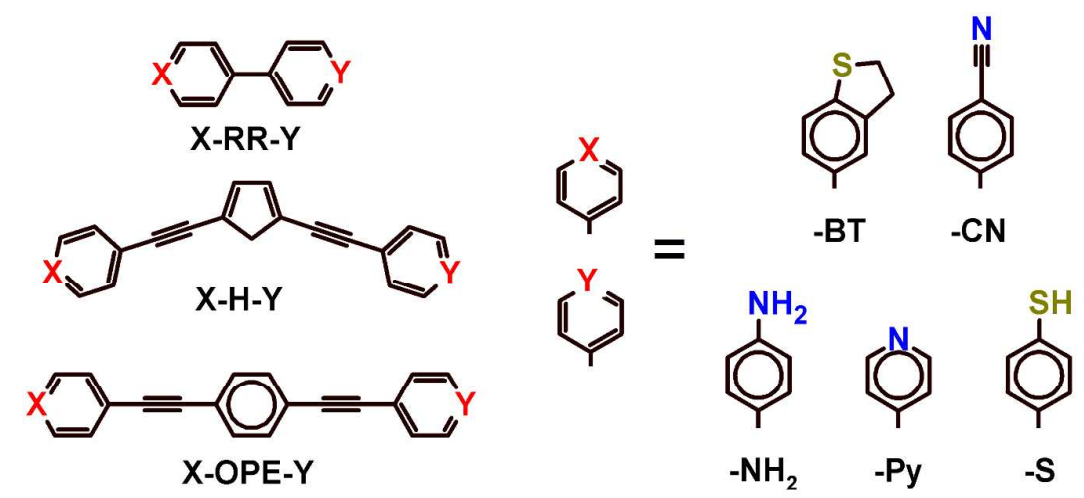

b)

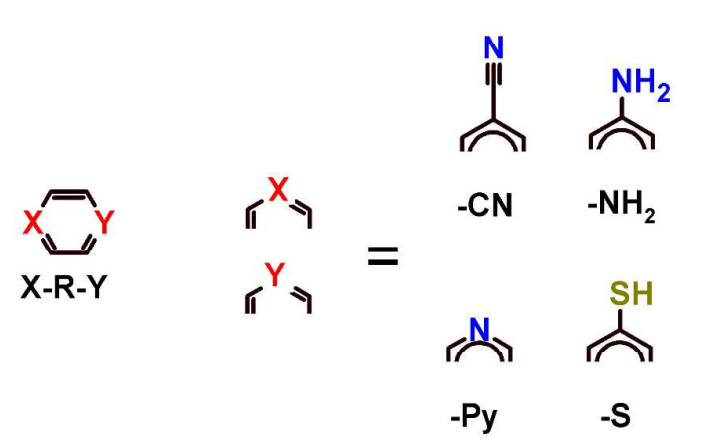

c)

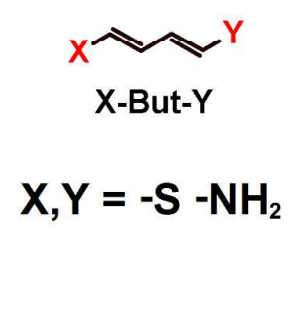

Figure 1. The 193 combinations of backbones and anchors used to obtain figure 2. a) The left column shows 3 alkanes, 3 alkenes and 3 alkynes (where $n=1,2,3$ ) and the second-from-left column shows 3 other backbones. (ie a total of 12 backbones). The right panel shows 5 anchors cyano $(\mathrm{CN})$, pyridil $(\mathrm{Py})$, dihidrbenzothiol $(\mathrm{BT})$, amine $\left(\mathrm{NH}_{2}\right)$ and thiol(S). These combine to yield $12 \times 5$ symmetric molecules and $12 \times 5 \times 4 / 2=120$ asymmetric molecules (ie 180 molecules in total). b) Molecules with a single ring as the backbone and four kinds of anchors: in total there are 4 symmetric and 6 asymmetric variants of these (ie 10 in total). c) shows a single butadiene chain with two kinds of anchors: in total there is one symmetric and 
two asymmetric versions of this. All of the molecular geometries are shown in the SI in Figures S1-S14.

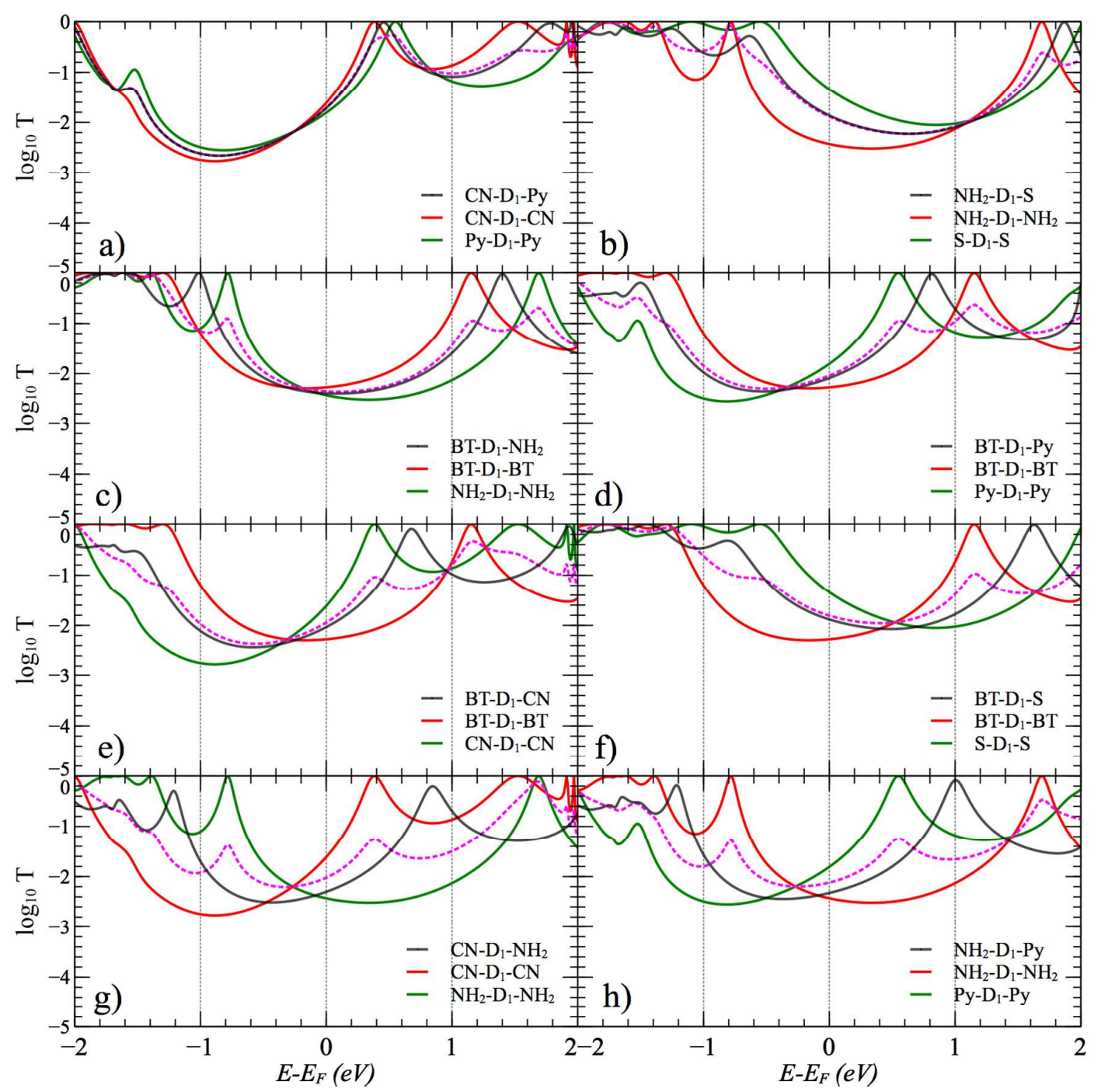

Figure 2. Transmission coefficients for symmetric and asymmetric molecules $\mathrm{X}-\mathrm{D}_{1}-\mathrm{X}, \mathrm{Y}-\mathrm{D}_{1}$ $\mathrm{Y}$ and $\mathrm{X}-\mathrm{D}_{1}-\mathrm{Y}$. The dashed pink curves show the circuit rule predictions for $\mathrm{X}-\mathrm{D}_{1}-\mathrm{Y}$. Choces of X, Y are: (a) CN, Py (b) $\mathrm{NH}_{2}, \mathrm{~S}$ (c) BT, $\mathrm{NH}_{2}$ (d) BT, Py (e) BT, CN (f) BT, S (g) CN, $\mathrm{NH}_{2}$ (h) $\mathrm{Py}, \mathrm{NH}_{2}$. Additional transmission coefficient curves are shown in the $\mathrm{SI}$ in Figures S15-S28. 

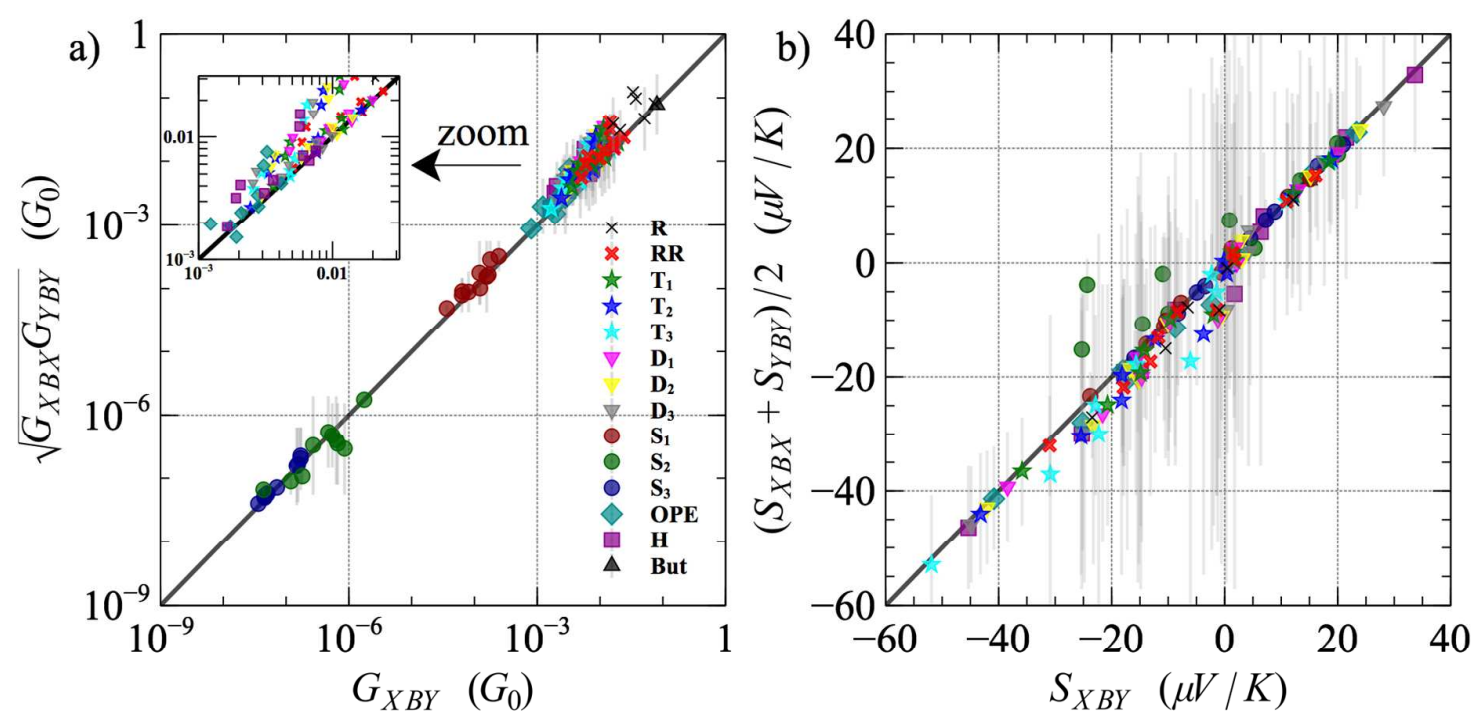

Figure 3. a) Comparison between the conductances $\mathrm{G}_{\mathrm{XBY}}$ and the geometric means $\left(G_{\mathrm{XBX}} G_{\mathrm{YBY}}\right)^{1 / 2}$. b) Comparison between the thermopowers $\mathrm{S}_{\mathrm{XBY}}$ and the arithmetic means $\left(S_{\mathrm{XBX}}+S_{\mathrm{YBY}}\right) / 2$. The top and bottom ends of the vertical grey lines show the values for the symmetric molecules used in the circuit rules. The different colours and markers indicate the various molecular backbones. The notation in the legend is as follows the backbone labelling in Figure 1. 

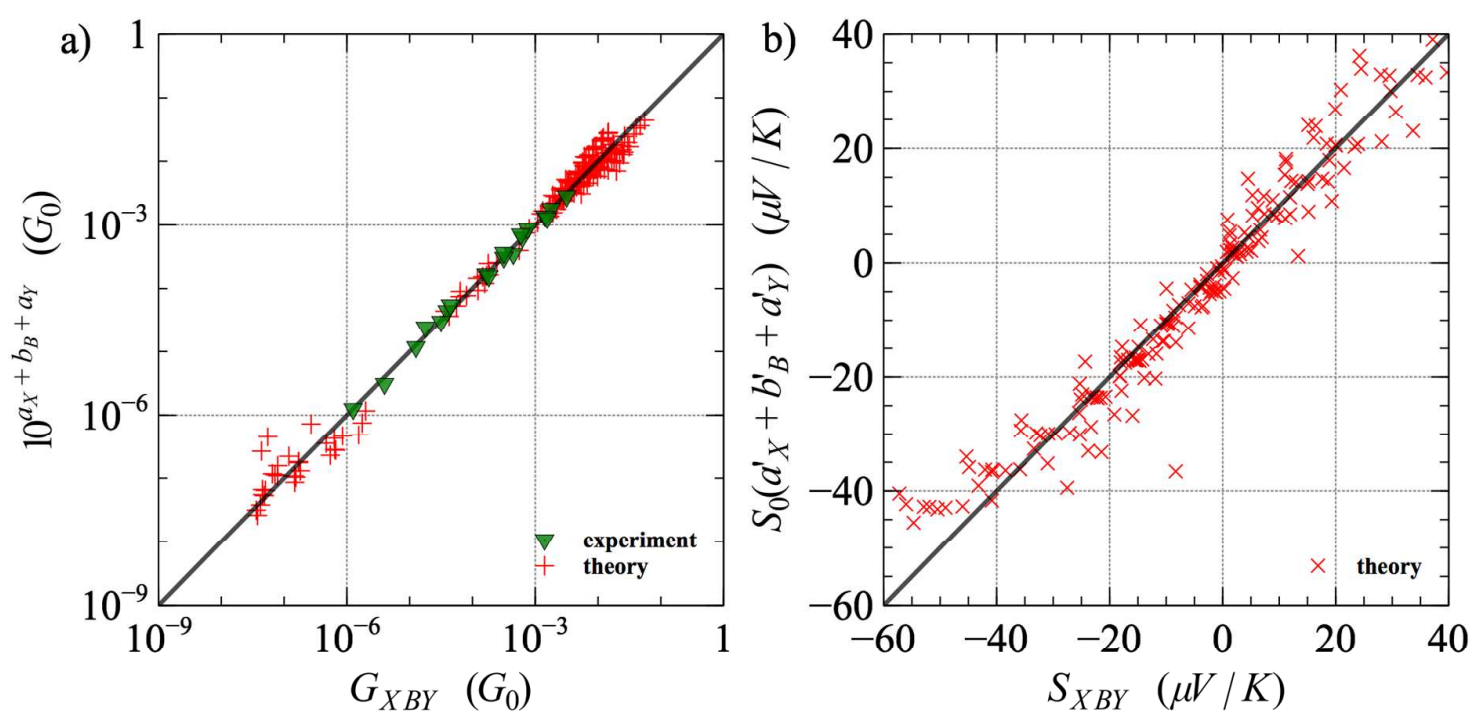

Figure 4. Factorized conductance and thermopower approximations are plotted against the DFT computed values (red markers) and experimental values (green triangles). In panel a), for each DFTcomputed conductance $G_{\mathrm{XBY}}$, a red cross marks the corresponding $10^{a_{\mathrm{X}}+b_{\mathrm{B}}+a_{\mathrm{Y}}} G_{0}$ value. In panel b) for each DFT-computed thermopower $S_{\mathrm{XBY}}$, a red cross marks the corresponding $\left(a_{\mathrm{X}}^{\prime}+b_{\mathrm{B}}^{\prime}+\right.$ $\left.a_{\mathrm{Y}}^{\prime}\right) S_{0}$ value. In both cases the $a_{\mathrm{X}}, a_{\mathrm{X}}^{\prime}$ parameters and $b_{\mathrm{B}}, b_{\mathrm{B}}^{\prime}$ parameters are taken from Table 1. The green triangles in panel a) show the factorization of a selection of experimental conductances from the literature, quoted in Table 3. For each experimental conductance $G_{\mathrm{XBY}}$ the green triangle marks the corresponding $10^{a_{\mathrm{X}}+b_{\mathrm{B}}+a_{\mathrm{Y}}} G_{0}$ value, where the $a_{\mathrm{X}}$ parameters and $b_{\mathrm{B}}$ parameters are taken from Table 2. $G_{0}=2 e^{2} / h . S_{0}=k_{B} / e$. 
a)

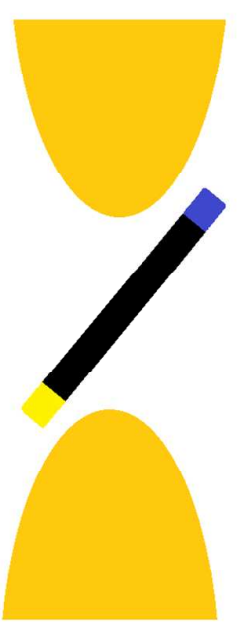

b)

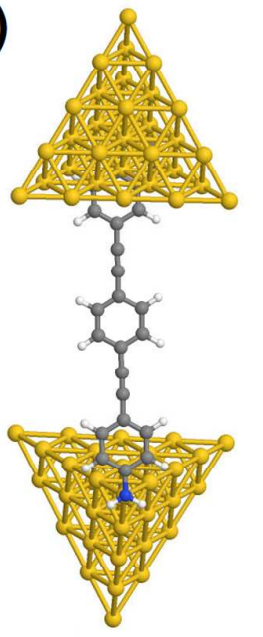

c)

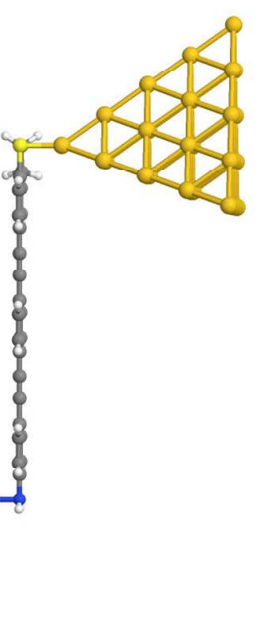

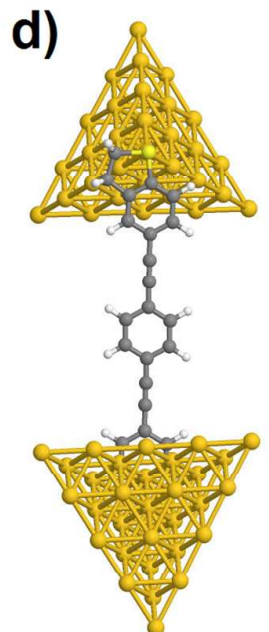

Figure 5. a) Molecule arrangement in break-junction experiment. b),c),d) Three views of the same junction. Top, side and bottom views, respectively, of the model junction geometry of a particular case (BT-OPE-NH 2$)$ for systematic comparisons.

\section{TABLES}

Table 1 Anchor and backbone parameters obtained by fitting eqs. 3 and 4 to the DFT-computed room temperature conductances $\left(\log _{10}\left(G / G_{0}\right)\right)$ and thermopowers $\left(S e / k_{B}\right)$ respectively

\begin{tabular}{|c|c|c|c|c|c|c|}
\hline & & & & \\
\hline & & $-\mathrm{BT}$ & $-\mathrm{CN}$ & $-\mathrm{NH}_{2}$ & $-\mathrm{Py}$ & $-\mathbf{S}$ \\
\hline$a_{\mathrm{X}}$ & & -1.12 & -0.89 & -1.20 & -0.87 & -0.68 \\
\hline$a_{\mathrm{X}}^{\prime}$ & & -0.02 & -0.24 & 0.06 & -0.17 & 0.19 \\
\hline Backbones B & $\mathrm{RR}$ & $\mathrm{T}_{1}$ & $\mathrm{~T}_{2}$ & $\mathrm{~T}_{3}$ & $\mathrm{OPE}$ & $\mathrm{H}$ \\
\hline$b_{\mathrm{B}}$ & 0.00 & -0.12 & -0.25 & -0.38 & -0.73 & -0.46 \\
\hline$b_{\mathrm{B}}^{\prime}$ & 0.00 & -0.01 & -0.05 & -0.09 & -0.02 & 0.01 \\
\hline Backbones B & $\mathrm{D}_{1}$ & $\mathrm{D}_{2}$ & $\mathrm{D}_{3}$ & $\mathrm{~S}_{1}$ & $\mathrm{~S}_{2}$ & $\mathrm{~S}_{3}$ \\
\hline$b_{\mathrm{B}}$ & -0.09 & -0.22 & -0.35 & -2.04 & -4.57 & -5.19 \\
\hline$b_{\mathrm{B}}^{\prime}$ & -0.01 & -0.01 & -0.01 & 0.03 & 0.06 & 0.10 \\
\hline
\end{tabular}

Table 2 Anchor and backbone parameters obtained by fitting equ. 3 to the experimental room temperature conductance $\left(\log _{10}\left(G / G_{0}\right)\right)$. For the $n_{A}=5$ anchor and $n_{B}=6$ backbone, the parameters give the 
conductance of $n_{\mathrm{A}}\left(n_{\mathrm{A}}+1\right) n_{\mathrm{B}} / 2=90$ molecules in the form of $G_{X B Y}=10^{a_{X}+b_{B}+a_{Y}} G_{0} \cdot{ }^{(a)}$ an example for this backbone is 'pmp' from ref ${ }^{20}$.

\begin{tabular}{|c|c|c|c|c|c|c|}
\hline \multirow{2}{*}{ Anchor X with rigns } & & & & \\
& & $-\mathrm{BT}$ & $-\mathrm{CN}$ & $-\mathrm{NH}_{2}$ & $-\mathrm{Py}$ & $-\mathbf{S}$ \\
\hline$a_{\mathrm{X}}$ (from experiments) & - & -1.12 & -2.15 & -1.44 & -1.58 & -1.22 \\
\hline Backbones B & $\mathrm{RR}$ & $\mathrm{T}_{1}$ & $\mathrm{~T}_{2}$ & $\mathrm{~T}_{4}$ & $\mathrm{OPE}$ & $\mathrm{OPE}($ meta) \\
\hline$b_{\mathrm{B}}$ (from experiments) & 0.00 & -0.31 & -0.63 & -1.20 & -1.37 & -2.75 \\
\hline
\end{tabular}

Table 3 Comparison between experimental conductances and the computed conductances from equ. 3 , using the fitted anchor and backbone parameters shown in table 2. Experimental values are taken from (a) from ref ${ }^{40}$ by averaging STM-BJ and MCBJ values, ${ }^{(b)}$ from ref ${ }^{31},{ }^{(c)}$ from ref ${ }^{20},{ }^{(d)}$ from ref ${ }^{26},{ }^{(e)}$ from ref $^{39},{ }^{(f)}$ from ref ${ }^{29}$.

\begin{tabular}{|c|c|c|c|c|c|}
\hline Molecule X B Y & $\begin{array}{c}\text { Experimental } \\
\qquad \log _{10} \frac{G}{G_{0}}\end{array}$ & $a_{\mathrm{X}}+b_{\mathrm{B}}+a_{\mathrm{Y}}$ & Molecule X B Y & $\begin{array}{l}\text { Experimental } \\
\qquad \log _{10} \frac{G}{G_{0}}\end{array}$ & $a_{\mathrm{X}}+b_{\mathrm{B}}+a_{\mathrm{Y}}$ \\
\hline $\mathrm{Py}_{1} \mathrm{Py}$ & $-3.35^{(a)}$ & -3.47 & $\mathrm{CN} \mathrm{T}_{1} \mathrm{CN}$ & $-4.75^{(a)}$ & -4.61 \\
\hline $\mathrm{Py} \mathrm{T}_{2} \mathrm{Py}$ & $-3.78^{(a)}$ & -3.79 & $\mathrm{CN} \mathrm{T}_{2} \mathrm{CN}$ & $-4.9^{(\mathrm{a})}$ & -4.93 \\
\hline $\mathrm{Py} \mathrm{T}_{4} \mathrm{Py}$ & $-4.4^{(\mathrm{a})}$ & -4.36 & $\mathrm{CN} \mathrm{T}_{4} \mathrm{CN}$ & $-5.4^{(\mathrm{a})}$ & -5.50 \\
\hline $\mathrm{NH}_{2} \mathrm{~T}_{1} \mathrm{NH}_{2}$ & $-3.205^{(a)}$ & -3.19 & $\mathrm{~S} \mathrm{~T}_{1} \mathrm{~S}$ & $-2.75^{(a)}$ & -2.75 \\
\hline $\mathrm{NH}_{2} \mathrm{~T}_{2} \mathrm{NH}_{2}$ & $-3.5^{(\mathrm{a})}$ & -3.51 & $\mathrm{~S} \mathrm{~T}_{2} \mathrm{~S}$ & $-3.12^{(a)}$ & -3.07 \\
\hline $\mathrm{BT} \mathrm{T}_{1} \mathrm{BT}$ & $-2.5^{(\mathrm{a})}$ & -2.55 & Py OPE Py & $-4.5^{(\mathrm{c})}$ & -4.53 \\
\hline $\mathrm{BT} \mathrm{T}_{2} \mathrm{BT}$ & $-2.845^{(a)}$ & -2.87 & S OPE S & $-3.74^{(d)}$ & -3.81 \\
\hline $\mathrm{BT} \mathrm{T}_{4} \mathrm{BT}$ & $-3.5^{(\mathrm{a})}$ & -3.44 & $\mathrm{NH}_{2} \mathrm{OPE} \mathrm{NH} \mathrm{NH}_{2}$ & $-4.35^{(\mathrm{e})}$ & -4.25 \\
\hline Py RR Py & $-3.23^{(b)}$ & -3.15 & $\begin{array}{l}\text { Py OPE(meta) Py } \\
\left(\mathrm{pmp}^{(\mathrm{c})}\right)\end{array}$ & $-5.9^{(\mathrm{c})}$ & -5.91 \\
\hline $\mathrm{NH}_{2} \mathrm{RR} \mathrm{NH}_{2}$ & $-2.81^{(\mathrm{f})}$ & -2.89 & & & \\
\hline
\end{tabular}




\section{ASSOCIATED CONTENT}

Supporting Information Available: Content includes figures for all molecular geometries and all computed transmission coefficient functions. Theoretical predictions for the conducance of all anchor group/backbone combinations are included in Table S1. This material is available free of charge via internet at http://pubs.acs.org.

\section{AUTHOR INFORMATION}

\section{Corresponding Author}

c.lambert@1ancaster.ac.uk; d.manrique@lancaster.ac.uk;

\section{Present Addresses}

†If an author's address is different than the one given in the affiliation line, this information may be included here.

\section{Author Contributions}


DZM, WH and CJL conceived the research idea. The calculations were performed by DZM and QA and the manuscript has been prepared by DZM, QA and CJL. The manuscript was written through contributions of all authors. All authors have given approval to the final version of the manuscript.

\section{Notes}

The authors declare no competing financial interest.

\section{ACKNOWLEDGMENTS}

This work was generously supported by the UK EPSRC projects EP/N017188/1, EP/M014452/1, the EC FP7 ITN “MOLESCO” project number 606728, and the Higher Education Ministry, Al Qadisiyah University, IRAQ.

\section{REFERENCES}

(1) Repp, J.; Meyer, G.; Paavilainen, S.; Olsson, F. E.; Persson, M. Science 2006, 312, (5777), 1196-1199.

(2) Donhauser, Z. J.; Mantooth, B. A.;Kelly, K. F.; Bumm, L. A.; Monnell, J. D.; Stapleton, J. J. ; Price Jr., D. W.; Rawlett, A. M.; Allara, D. L. Tour, J. M.; Weiss, P. S. Science 2001, 292, 2303-2307.

(3) Wold, D. J.; Haag, R.; Rampi, M. A.; Frisbie, C. D. J. Phys. Chem. B 2002, 106, (11), 2813-2816.

(4) Fan, F. R. F. J. Am. Chem. Soc. 2002, 124, 5550-5560.

(5) Widawsky, J. R.; Darancet, P.; Neaton, J. B.; Venkataraman, L. Nano Lett. 2012, 12, (1), 354-358.

(6) Venkataraman, L.; Klare, J. E.; Nuckolls, C.; Hybertsen, M. S.; Steigerwald, M. L. Nat. 2006, 442, (7105), 904-907.

(7) Hong, W.; Valkenier, H.; Meszaros, G.; Manrique, D. Z.; Mishchenko, A.; Putz, A.; Garcia, P. M.; Lambert, C. J.; Hummelen, J. C.; Wandlowski, T. Beilstein J. Nanotechnol. 2011, 2, 699-713.

(8) Seferos, D. S.; Trammell, S. A.; Bazan, G. C.; Kushmerick, J. G. P. NATL. ACAD. SCI. USA 2005, 102, (25), 8821-8825.

(9) Dadosh, T.; Gordin, Y.; Krahne, R.; Khivrich, I.; Mahalu, D.; Frydman, V.; Sperling, J.; Yacoby, A.; Bar-Joseph, I. Nat. 2005, 436, (7054), 1200-1200.

(10) Liao, J.; Bernard, L.; Langer, M.; Schonenberger, C.; Calame, M. Adv. Mater. 2006, 18, (18), 2803-2804.

(11) Reed, M. A.; Zhou, C.; Muller, C. J.; Burgin, T. P.; Tour, J. M. Science 1997, 278, (5336), 252-254.

(12) Park, J.; Pasupathy, A. N.; Goldsmith, J. I.; Chang, C.; Yaish, Y.; Petta, J. R.; Rinkoski, M.; Sethna, J. P.; Abruña, H. D.; McEuen, P. L. Nat. 2002, 417, (6890), 722-725.

(13) Osorio, E.; Bjørnholm, T.; Lehn, J.; Ruben, M.; Van der Zant, H. J. Phys.: Condens. Matter 2008, 20, (37), 374121.

(14) Chen, J.; Reed, M. A.; Rawlett, A. M.; Tour, J. M. Science 1999, 286, 1550-1552. 
(15) Aviram, A.; Ratner, M. Chem. Phys. Lett. 1974, 29, 277-283.

(16) Finch, C. M.; Sirichantaropass, S.; Bailey, S. W.; Grace, I. M.; García-Suárez, V. M.; Lambert, C. J. J. Phys.: Condens. Matter 2008, 20, (2), 022203.

(17) Peterfalvi, C. G.; Grace, I.; Manrique, D. Z.; Lambert, C. J. J. Chem. Phys 2014, 140, (17), 174711.

(18) Evangeli, C.; Gillemot, K.; Leary, E.; González, M. T.; Rubio-Bollinger, G.; Lambert, C. J.; Agrait, N. Nano Lett. 2013, 13, (5), 2141-2145.

(19) Zhao, X.; Huang, C.; Gulcur, M.; Batsanov, A. S.; Baghernejad, M.; Hong, W.; Bryce, M. R.; Wandlowski, T. Chem. Mater. 2013, 25, (21), 4340-4347.

(20) Manrique, D. Z.; Huang, C.; Baghernejad, M.; Zhao, X.; Al-Owaedi, O. A.; Sadeghi, H.; Kaliginedi, V.; Hong, W.; Gulcur, M.; Wandlowski, T.; Bryce, M. R.; Lambert, C. J. Nat. Commun. 2015, 6, 6389A, No. 8.

(21) Lambert, C. Chem. Soc. Rev. 2015, 44, (4), 875-888.

(22) Cuevas, J. C.; Scheer, E., Molecular electronics: an introduction to theory and experiment. World Scientific: 2010; Vol. 1.

(23) Sparks, R.; García-Suárez, V. M.; Manrique, D. Z.; Lambert, C. J. Phys. Rev. B 2011, 83, (7), 075437.

(24) Finch, C. M.; Garcia-Suarez, V. M.; Lambert, C. J. Phys. Rev. B 2009, 79, (3), 033405 .

(25) Hong, W.; Manrique, D. Z.; Moreno-Garcia, P.; Gulcur, M.; Mishchenko, A.; Lambert, C. J.; Bryce, M. R.; Wandlowski, T. J. Am. Chem. Soc. 2012, 134, (4), 2292-2304.

(26) Kaliginedi, V.; Moreno-García, P.; Valkenier, H.; Hong, W.; García-Suárez, V. M.; Buiter, P.; Otten, J. L. H.; Hummelen, J. C.; Lambert, C. J.; Wandlowski, T. J. Am. Chem. Soc. 2012, 134, (11), 5262-5275.

(27) Yoshida, K.; Pobelov, I. V.; Manrique, D. Z.; Pope, T.; Meszaros, G.; Gulcur, M.; Bryce, M. R.; Lambert, C. J.; Wandlowski, T. Sci. Rep. 2015, 5, 9002A, No. 8.

(28) Mishchenko, A.; Zotti, L. A.; Vonlanthen, D.; Buerkle, M.; Pauly, F.; Carlos Cuevas, J.; Mayor, M.; Wandlowski, T. J. Am. Chem. Soc. 2011, 133, (2), 184-187.

(29) Hybertsen, M. S.; Venkataraman, L.; Klare, J. E.; Cwhalley, A.; Steigerwald, M. L.; Nuckolls, C. J. Phys.: Condens. Matter 2008, 20, (37), 374115.

(30) Quek, S. Y.; Venkataraman, L.; Choi, H. J.; Louie, S. G.; Hybertsen, M. S.; Neaton, J. B. Nano Lett. 2007, 7, (11), 3477-3482.

(31) Quek, S. Y.; Kamenetska, M.; Steigerwald, M. L.; Choi, H. J.; Louie, S. G.; Hybertsen, M. S.; Neaton, J. B.; Venkataraman, L. Nat. Nanotechnol. 2009, 4, (4), 230-234.

(32) Kanthasamy, K.; Pfnuer, H. Beilstein J. Nanotechnol. 2015, 6, 1690-1697.

(33) Egger, D. A.; Liu, Z.-F.; Neaton, J. B.; Kronik, L. Nano Letters 2015, 15, (4), 24482455.

(34) Jin, C.; Strange, M.; Markussen, T.; Solomon, G. C.; Thygesen, K. S. J. Chem. Phys. 2013, 139, (18), 184307.

(35) Quek, S. Y.; Khoo, K. H. Acc. Chem. Res. 2014, 47, (11), 3250-3257.

(36) Berritta, M.; Manrique, D. Z.; Lambert, C. J. Nanoscale 2015, 7, (3), 1096-1101.

(37) Wang, K.; Zhou, J.; Hamill, J. M.; Xu, B. J. Chem. Phys. 2014, 141, (5), 054712.

(38) Baghernejad, M.; Manrique, D. Z.; Li, C.; Pope, T.; Zhumaev, U.; Pobelov, I.; Moreno-Garcia, P.; Kaliginedi, V.; Huang, C.; Hong, W.; Lambert, C.; Wandlowski, T. Chem. Commun. 2014, 50, (100), 15975-15978.

(39) Gonzalez, T. M.; Zhao, X.; Manrique, D. Z.; Miguel, D.; Leary, E.; Gulcur, M.; Batsanov, A. S.; Rubio-Bollinger, G.; Lambert, C. J.; Bryce, M. R.; Agrait, N. J. Phys. Chem. C 2014, 118, (37), 21655-21662. 
(40) Moreno-Garcia, P.; Gulcur, M.; Manrique, D. Z.; Pope, T.; Hong, W.; Kaliginedi, V.; Huang, C.; Batsanov, A. S.; Bryce, M. R.; Lambert, C.; Wandlowski, T. J. Am. Chem. Soc. 2013, 135, (33), 12228-12240.

(41) Kim, T.; Darancet, P.; Widawsky, J. R.; Kotiuga, M.; Quek, S. Y.; Neaton, J. B.; Venkataraman, L. Nano Lett. 2014, 14, (2), 794-798.

(42) Sedghi, G.; García-Suárez, V. M.; Esdaile, L. J.; Anderson, H. L.; Lambert, C. J.; Martín, S.; Bethell, D.; Higgins, S. J.; Elliott, M.; Bennett, N. Nat. Nanotechnol. 2011, 6, (8), 517-523.

(43) Sangtarash, S.; Huang, C.; Sadeghi, H.; Sorohhov, G.; Hauser, J. r.; Wandlowski, T.; Hong, W.; Decurtins, S.; Liu, S.-X.; Lambert, C. J. J. Am. Chem. Soc 2015, 137, (35), $11425-$ 11431.

(44) Geng, Y.; Sangtarash, S.; Huang, C.; Sadeghi, H.; Fu, Y.; Hong, W.; Wandlowski, T.; Decurtins, S.; Lambert, C. J.; Liu, S.-X. J. Am. Chem. Soc 2015, 137, (13), 4469-4476.

(45) Stewart, J. J. P. J. Mol. Model. 2013, 19, (1), 1-32.

(46) Huang, C. C.; Rudnev, A. V.; Hong, W. J.; Wandlowski, T. Chem. Soc. Rev. 2015, 44, (4), 889-901.

(47) Soler, J. M.; Artacho, E.; Gale, J. D.; García, A.; Junquera, J.; Ordejón, P.; SánchezPortal, D. J. Phys.: Condens. Matter. 2002, 14, (11), 2745.

(48) Ferrer, J.; Lambert, C. J.; Garcia-Suarez, V. M.; Manrique, D. Z.; Visontai, D.; Oroszlany, L.; Rodriguez-Ferradas, R.; Grace, I.; Bailey, S. W. D.; Gillemot, K.; Sadeghi, H.; Algharagholy, L. A. New J. Phys. 2014, 16, 093029.

(49) Verzijl, C. J. O.; Seldenthuis, J. S.; Thijssen, J. M. J. Chem. Phys. 2013, 138, (9), 094102 .

\section{TABLE OF CONTENT GRAPHICS}

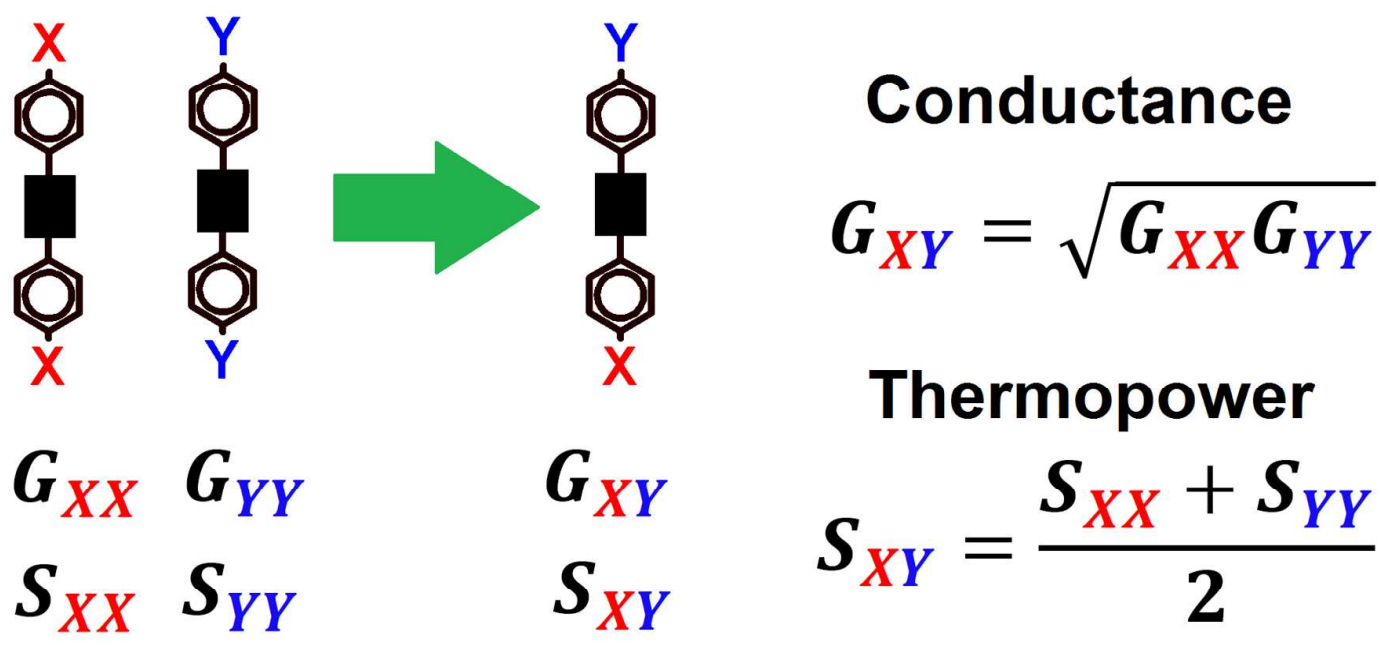


TABLE OF CONTENT GRAPHICS

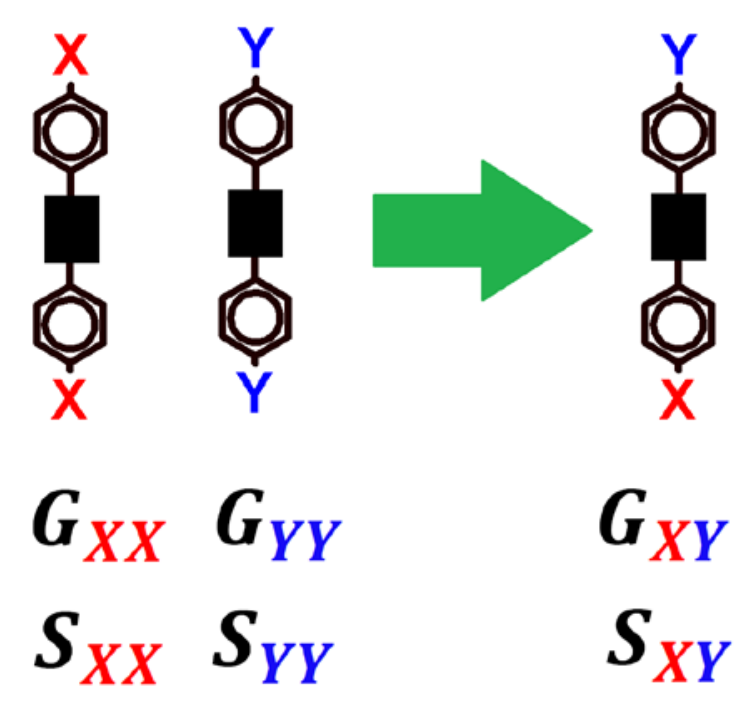

\section{Conductance}

$\boldsymbol{G}_{X Y}=\sqrt{\boldsymbol{G}_{X X} \boldsymbol{G}_{Y Y}}$

Thermopower 